\title{
Molecules for the origin of life from impact-generated atmospheres on early Earth
}

\author{
NICHOLAS WOGAN ${ }^{1}$, DAVID C. CATLING ${ }^{1}$ AND KEVIN \\ ZAHNLE $^{2}$
}

${ }^{1}$ University of Washington

${ }^{2}$ NASA Ames Research Center

Presenting Author: wogan@uw.edu

While there are several theories for the origin of life, the presence of ribonucleic molecular "fossils" in modern biology has led to the RNA World hypothesis. This hypothesis proposes a stage of primitive life with RNA as a self-replicating molecule that evolved by natural selection, which, at some point, was encapsulated in a cell. RNA needs to be produced abiotically on early Earth in this scenario. Chemists have proposed several schemes, but all pathways require nitriles - hydrogen cyanide $(\mathrm{HCN})$, cyanoacetylene $(\mathrm{HCCCN})$, and cyanogen $(\mathrm{NCCN})$ - to synthesize nucleobases, which are building blocks of RNA.

Abiotic synthesis of nitriles very likely requires a reduced prebiotic atmosphere, i.e., one redox-dominated by hydrogenbearing reducing gases, and consisting of, e.g., $\mathrm{H}_{2}, \mathrm{CH}_{4}$ with $\mathrm{CO}_{2}$ or $\mathrm{CO}$, and $\mathrm{N}_{2}$ and/or $\mathrm{NH}_{3}$. Photochemistry makes $\mathrm{HCN}$ from the nitrogenous gases only if $\mathrm{CH}_{4}$ is abundant. Also, $\mathrm{HCCCN}$ and NCCN are produced in highly reducing atmospheres, e.g. Titan's.

However, geochemical evidence suggests that volcanoes did not produce very reduced gases in the Hadean, and abundant volcanic $\mathrm{CH}_{4}$, in particular, was unlikely. Instead, impacts are a plausible source.

Asteroid impacts would make a reducing atmosphere because reactions between iron-rich impact ejecta and shock-heated water vapor from the ocean would generate substantial amounts of $\mathrm{H}_{2}$, $\mathrm{CH}_{4}$ and $\mathrm{NH}_{3}$. Subsequent photochemistry would have generated prebiotic molecules like HCN making a "window of opportunity" for abiotic synthesis and evolution of RNA.

Here, we simulate creation and evolution of impact-generated reducing atmospheres with a novel 1-dimensional coupled climate-photochemical model, improving upon previous work (1). We quantify the surface deposition prebiotic nitriles following impacts with diameters between 2700 ( Pluto-size) and $20 \mathrm{~km}$. We combine our modeling results with estimates of the impact flux on the early Earth to calculate the surface temperature and surface deposition flux of prebiotic relevant nitriles during the first billion years of Earth's history.

1. Zahnle, Lupu, Catling \& Wogan (2020). Creation and evolution of impact-generated reduced atmospheres of early Earth. Planetary Science Journal. doi:10.3847/PSJ/ab7e2c. 